\title{
VOZ E DISFUNÇÃO TEMPOROMANDIBULAR EM PROFESSORES
}

\section{Voice and temporomandibular joint disorders in teachers}

\author{
Ilza Maria Machado (1), Esther Mandelbaum Gonçalves Bianchini ${ }^{(2)}$, \\ Marta Assumpção de Andrada e Silva ${ }^{(3)}$, Léslie Piccolotto Ferreira ${ }^{(4)}$
}

\section{RESUMO}

Objetivo: verificar a presença e possível correlação entre alteração vocal e DTM, em professores, a partir de dados de avaliação autorreferida, fonoaudiológica, otorrinolaringológica e odontológica. Métodos: participaram deste estudo, 29 professores de uma escola de rede pública do ensino fundamental e médio do município de Sorocaba - SP. Os professores responderam questionário para levantamento de alteração vocal, e de disfunção temporomandibular (DTM). Foram realizadas quatro avaliações: perceptivo-auditiva; otorrinolaringológica; motricidade orofacial e odontológica. A menção a três ou mais sintomas no questionário determinou "presença" de queixa de voz e de DTM. As avaliações: perceptivo-auditiva e otorrinolaringológica concluíram a "ausência" e "presença" de alteração de voz e de laringe. Nas avaliações da motricidade orofacial e odontológica foi considerada DTM quando registrados três ou mais sinais e/ou sintomas, sendo indispensável à presença de dor. $\mathrm{Na}$ análise estatística dos dados, foram empregados: teste de Igualdade de Duas Proporções, teste exato de Fisher e de concordância Kappa. Resultados: dentre os participantes, 82,8\% fizeram autorreferência à alteração vocal e $62,1 \%$ de sintomas de DTM; $51,7 \%$ apresentaram alteração de voz na avaliação otorrinolaringológica e $65,5 \%$, alteração de DTM na avaliação odontológica. Na comparação da avaliação de alteração de voz e DTM foi registrada correlação significante presente na avaliação perceptivo-auditiva da voz e de motricidade orofacial para DTM, e com tendência a significância na aplicação do questionário. Conclusão: os resultados apontam na direção de confirmar a presença de alteração de voz e DTM no grupo de professores pesquisado e correlação entre os mesmos.

DESCRITORES: Voz; Distúrbios da Voz; Docentes; Síndrome da Disfunção da Articulação Temporomandibular; Dor Facial

(1) Fonoaudióloga; Mestre em Fonoaudiologia pela Pontifícia Universidade Católica de São Paulo.

(2) Fonoaudióloga; Professora Adjunta Programa de Pós-Graduação Mestrado Profissional em Fonoaudiologia Universidade Veiga de Almeida, UVA, Rio de Janeiro, RJ; Professora do CEFAC - Pós-Graduação em Saúde e Educação, São Paulo, SP; Doutora em Ciências - Fisiopatologia Experimental pela Faculdade de Medicina da Universidade de São Paulo.

(3) Fonoaudióloga; Professora Assistente Doutora no Programa de Estudos Pós-Graduados e no Curso de Fonoaudiologia da Pontifícia Universidade Católica, PUC, São Paulo, SP; Professora Adjunta do Curso de Fonoaudiologia da Faculdade de Ciências Médicas da Santa Casa de São Paulo, FCMSCSP, São Paulo, SP; Professora nos Cursos de Especialização em Voz da Coordenadoria Geral de Especialização, Aprimoramento e Extensão da Pontifícia Universidade Católica de São Paulo, COGEAE/PUC-SP, São Paulo, SP; e do CEFAC - Pós-Graduação em Saúde e Educação, São Paulo, SP; Doutora em Comunicação e Semiótica pela Pontifícia Universidade Católica de São Paulo.

(4) Fonoaudióloga; Professora Titular do Departamento de Fundamentos da Fonoaudiologia e do Programa de

\section{INTRODUÇÃO}

O professor se enquadra em uma categoria profissional, na qual a voz é considerada um dos principais instrumentos de trabalho, e, portanto está predisposto a desenvolver alterações vocais.

De acordo com a literatura, as alterações vocais surgem em função de diferentes fatores: os denominados organizacionais, ambientais e biológicos ${ }^{1-11}$.

Estudos Pós-Graduados em Fonoaudiologia da Pontifícia Universidade Católica de São Paulo, PUC, São Paulo, SP; Coordenadora e Docente do Curso de Especialização em Fonoaudiologia - Voz - da Coordenadoria Geral de Especialização, Aprimoramento e Extensão da Pontifícia Universidade Católica de São Paulo, COGEAE/PUC-SP, São Paulo, SP; Doutora em Distúrbios da Comunicação Humana pela Universidade Federal de São Paulo - Escola Paulista de Medicina.

Conflito de interesses: inexistente 
No que se refere às disfunções temporomandibulares (DTMs), essas também estão presentes entres as queixas de professores e, a princípio, as relações entre alterações vocais e DTMs envolveriam, principalmente, o desequilíbrio funcional da musculatura extrínseca da laringe ocasionado pelas restrições dos movimentos mandibulares durante a fala ${ }^{6,7}$.

Importante lembrar que esses distúrbios são resultantes ainda, do desequilíbrio da musculatura orofacial, cervical, supralaríngea e da modulação dos mecanismos neuromusculares periféricos e centrais, conforme comprovado em diferentes trabalhos ${ }^{12-16}$.

As DTMs são caracterizadas por uma série de sinais e sintomas clínicos que se manifestam, como: dores musculares, cefaleia, estalidos na articulação temporomandibular (ATM), dificuldades em realizar movimentos mandibulares e redução dos movimentos mandibulares durante a fala ${ }^{17-24}$. Na presença de DTM, o principal sintoma é dor na ATM, tanto na posição em repouso como no movimento de abertura mandibular ${ }^{25}$.

Ao longo dos últimos dez anos, pesquisas têm evidenciado a relação entre sintomatologia de DTM e dificuldade ao falar, principalmente referente aos movimentos mandibulares durante a fonação ${ }^{26-29}$.

A redução dos movimentos da amplitude e consequente articulação da fala mais travada leva ao comprometimento da emissão vocal, devido à modificação das caixas de ressonância. Os ajustes compensatórios, como redução da cavidade oral e tensão excessiva na região orofacial, são responsáveis por uma fonação com esforço e consequente queixa de cansaço, dor e desconforto ao falar muito, o que pode desencadear alteração vocal ${ }^{26}$.

Uma pesquisa ${ }^{30}$ demonstrou que sujeitos com queixas e sintomatologia de DTM têm maior tendência em apresentar alterações vocais ${ }^{30}$. Nos resultados dessa pesquisa ${ }^{30}$, que envolveu 43 pessoas, estiveram presentes sintomas de rouquidão $(24,96 \%)$, seguidos de soprosidade $(8,32 \%)$ e aspereza (4,17\%). O estudo mostrou significância estatística para alteração da ressonância da voz, pitch e loudness.

Outro estudo mostrou que a relação entre alteração e voz e DTM está diretamente relacionada ao grau de sintomatologia da DTM, quanto maior o grau de severidade da sintomatologia de DTM maior a influência desta no loudness e na ressonância da voz ${ }^{31}$.

O objetivo do presente estudo foi verificar a presença e possível correlação entre alteração vocal e DTM, em professores, a partir de dados de avaliação autorreferida, fonoaudiológica, otorrinolaringológica e odontológica.

\section{MÉTODOS}

Esta pesquisa é de natureza transversal e observacional. Os participantes do estudo assinaram termo de consentimento livre e esclarecido.

O estudo foi desenvolvido por uma equipe multidisciplinar, composta por um médico otorrinolaringologista, duas fonoaudiólogas e um odontólogo (especialista em cirurgia bucomaxilofacial).

Concordaram em tomar parte do estudo 45 professores. Desses, 16 foram excluídos, pois não participaram da etapa inicial da amostra, momento em que a qualidade vocal e laríngea foram avaliadas. Assim, o estudo foi composto por 29 participantes, 19 do sexo feminino e 10 do sexo feminino, com média de 43,21 anos.

Os instrumentos e procedimentos para o desenvolvimento do estudo foram os seguintes:

1. Questionário autorreferido que contou com 15 questões referentes a alterações vocais ${ }^{32}$, e queixas relacionadas aos sintomas de DTM ${ }^{26}$ (Figura 1).

2. Protocolo para coleta de amostra de fala para avaliar a qualidade vocal. Foi solicitado a cada professor que emitisse a vogal /a/ sustentada e em escala (ascendente e descendente) e, a seguir, simulasse um trecho de uma aula com a temática de sua escolha, sem tempo determinado, (Figura 2).

3. Exame de videonasolaringoscopia, para investigar a presença ou ausência de alteração laríngea. Para a realização do exame de laringe, o médico, otorrinolaringologista, solicitou que o professor emitisse /a/, /e/ e /i/, de forma sustentada; /a/ ou /e/, em escala ascendente, conforme a facilidade de execução; /a/, em fonação inspirada; e, por fim, contagem de 1 (um) a 10 (dez) ${ }^{33}$, que analisa a presença de: constrição supra-glótica (constrição medial ou ântero-posterior), lesão de massa em pregas vocais (nódulos, pólipo, edema de Reinke, espessamento, edema e cisto), presença de sinais de refluxo gastroesofágico e fendas (Figura 3).

4. Avaliação odontológica: protocolo para averiguar sinais de DTM, elaborado para esta pesquisa, levantou dados referentes à presença de DTM, por meio da verificação de mialgia, sinais clínicos de DTM (crepitação, estalido, limitação do movimento mandibular) e presença de hábitos deletérios (bruxismo, apertar os dentes e onicofagia) (Figura 4).

5. Avaliação da motricidade orofacial ${ }^{26}$ : foram analisados sinais de DTM, por meio da verificação de simetria da musculatura, sinais na mucosa jugal, na língua e simetria dos movi- 
mentos mandibulares. Verificou-se, por meio de inspeção manual, a presença de assimetria e contração dos músculos temporais, masseteres, bucinadores e mentual. Os movimentos mandibulares foram avaliados quanto à amplitude, desvios do percurso, limitações, ruídos e dor durante movimento. No movimento de abertura, a amplitude de boca foi verificada, por meio de paquímetro digital, modelo DIGIMERS
Pró-Fono-174BL, e a mensuração registrada em milímetros. Considerou-se como valor de referência de amplitude para abertura de boca, o mínimo de $40 \mathrm{~mm}$ baseados em estudos anteriores ${ }^{26,34}$. Em seguida, solicitou-se ao professor abrir e fechar a boca, lateralizar a mandíbula para direita e para esquerda e protruí-la, verificando-se a habilidade em realizar o movimento (Figura 5).

\section{VOZ E DISFUNÇÃO TEMPOROMANDIBULAR}

Prezado professor: O questionário abaixo tem como objetivo fazer um levantamento das condições vocais e da articulação temporomandibular do professor. Por gentileza, responda todas as questões, fazendo um $\underline{x}$ no local indicado ou completando, quando solicitado.

\section{I - IDENTIFICAÇÃO DO QUESTIONÁRIO}

\begin{tabular}{|l|l|l|}
\hline 1 & Código do entrevistado (não preencher): \\
\hline 2 & Data: / / / \\
\hline
\end{tabular}

\section{II - IDENTIFICAÇÃO DO ENTREVISTADO}

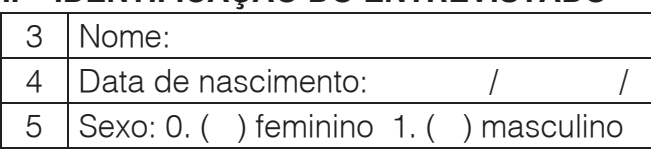

\section{III - ASPECTOS VOCAIS}

6 Você tem ou já teve alteração na sua voz?

0. ( ) não 1. ( ) sim, tive 2. ( ) sim, tenho

7 Se você tem alteração na voz, há quanto tempo esta alteração está presente?

1. ( ) 0 a 5 meses 2. ( ) 6 meses a 11 meses 3. ( ) 1 a 2 anos 4. ( ) 3 a 4 anos 5. ( ) mais de 4 anos

8 Se você teve/tem alteração de voz, em sua opinião, o que a causou:

\begin{tabular}{|l|l|}
\hline 1. ( ) uso intensivo da voz & $6 .($ ) exposição ao frio \\
\hline 2. ( ) infecção respiratória & $7 .($ ) exposição ao barulho \\
\hline 3. ( ) alergia & 8. ( ) não houve causa aparente \\
\hline 4. ( ) estresse & $9 .($ ) não sei \\
\hline 5. ( ) gripe constante & 10. ( ) outros, qual/quais? \\
\hline
\end{tabular}

9 Quais sintomas vocais você tem atualmente?

\begin{tabular}{l|l}
\hline 1. rouquidão & 0. ( ) não 1. ( ) sim
\end{tabular}

\begin{tabular}{l|l} 
2. perda da voz & 0. ( ) não 1. ( ) sim
\end{tabular}

\begin{tabular}{l|l} 
3. falha na voz & 0. ( ) não 1. ( ) sim
\end{tabular}

\begin{tabular}{l|l} 
4. falta de ar & $0 .($ ) não 1. ( ) sim
\end{tabular}

\begin{tabular}{l|l}
\hline 5. voz fina & 0. ( ) não 1. ( ) sim
\end{tabular}

\begin{tabular}{l|l}
\hline 6. voz grossa & 0. ( ) não 1. ( ) sim
\end{tabular}

\begin{tabular}{l|l|l}
\hline 7. voz variando grossa / fina & 0. ( ) não 1. ( ) sim
\end{tabular}

\begin{tabular}{l|l} 
8. voz fraca & 0. ( ) não 1. ( ) sim
\end{tabular}

\begin{tabular}{l|l} 
9. outros & 0. ( ) não 1. ( ) sim
\end{tabular}

Quais?

Figura 1 - Questionário 


\begin{tabular}{|c|c|c|}
\hline \multirow[t]{15}{*}{10} & \multicolumn{2}{|c|}{ Quais sensações relacionadas à garganta e à voz (laringo-faríngeas) você tem, atualmente? } \\
\hline & 1. picada na garganta & 0. ( ) não 1. ( ) sim \\
\hline & 2. areia na garganta & 0. ( ) não 1. ( ) sim \\
\hline & 3. bola na garganta & 0. ( ) não 1.( ) sim \\
\hline & 4. pigarro & 0. ( ) não 1.( ) sim \\
\hline & 5. tosse seca & 0. ( ) não 1.( ) sim \\
\hline & 6. tosse com catarro & 0. ( ) não 1.( ) sim \\
\hline & 7. dor ao falar & 0. ( ) não 1. ( ) sim \\
\hline & 8. dor ao engolir & 0. ( ) não 1.( ) sim \\
\hline & 9. dificuldade para engolir & 0. ( ) não 1.( ) sim \\
\hline & 10. ardor na garganta & 0. ( ) não 1.( ) sim \\
\hline & 11.secreção/ catarro na garganta & 0. ( ) não 1.( ) sim \\
\hline & 12. garganta seca & 0. ( ) não 1.( ) sim \\
\hline & 13. cansaço ao falar & 0. ( ) não 1.( ) sim \\
\hline & 14. esforço ao falar & 0. ( ) não 1.( ) sim \\
\hline
\end{tabular}

\section{IV - SINTOMAS DE DISFUNÇÃO TEMPOROMANDIBULAR}

\begin{tabular}{|c|c|}
\hline 11 & \begin{tabular}{|l|ll} 
Dor de cabeça ou na face & $0 .($ ) não 1. ( ) sim \\
\end{tabular} \\
\hline 12 & $\begin{array}{l}\text { Em que situação essa dor ocorre? } \\
\text { ( ) ao acordar ( ) no final do dia ( ) ao mastigar ( ) ao falar muito ( ) ao abrir muito a boca } \\
\text { ( )independente do que estiver fazendo }\end{array}$ \\
\hline 13 & $\begin{array}{l}\text { Como você classificaria essa dor? } \\
\begin{array}{lll}(\mathrm{)}) \text { ausente }(0) & (\mathrm{l}) \text { leve }(1) & (\mathrm{l}) \text { moderada (2) }\end{array}\end{array}$ \\
\hline 14 & $\begin{array}{l}\text { Apresenta barulho como "estalos" ao abrir e fechar a boca? } \\
\text { 0. ( ) não 1.( ) sim }\end{array}$ \\
\hline 15 & $\begin{array}{l}\text { Apresenta barulho como ruído de "amassar papel" ou de "areia" ao abrir e fechar a boca? } \\
\text { O. ( ) não } 1 .(\text { ) sim }\end{array}$ \\
\hline 16 & $\begin{array}{l}\text { Apresenta limitação ou dificuldade em movimentar livremente a mandíbula? } \\
\text { O. ( ) não 1.( ) sim }\end{array}$ \\
\hline 17 & $\begin{array}{l}\text { Já apresentou algum episódio de } \\
\text { 0. ( ) não 1.( ) sim }\end{array}$ \\
\hline
\end{tabular}

Gostaria de acrescentar algum comentário?

(adaptado de Bianchini, 2000 e Ferreira et al., 2007)

Figura 1 - Questionário (continuação) 


\begin{tabular}{|c|c|c|c|c|c|c|}
\hline $\begin{array}{l}\text { Utilizar para } \\
\begin{array}{l}0=\text { normal } \\
1=\text { alteraçã } \\
2=\text { alteraçãã } \\
3=\text { alteraçã }\end{array}\end{array}$ & $\begin{array}{l}\text { la um dos } \\
\text { sente } \\
\text { screta } \\
\text { sderada } \\
\text { vera }\end{array}$ & Esce & la GIRBAS & & & \\
\hline & & & Parân & etros & & \\
\hline Professores & $\begin{array}{l}\text { Grau de } \\
\text { alteração } \\
\text { vocal }\end{array}$ & Instabilidade & Rugosidade & Soprosidade & Astenia & Tensão \\
\hline 1 & & & & & & \\
\hline 2 & & & & & & \\
\hline 3 & & & & & & \\
\hline 4 & & & & & & \\
\hline 5 & & & & & & \\
\hline 6 & & & & & & \\
\hline 7 & & & & & & \\
\hline 8 & & & & & & \\
\hline 9 & & & & & & \\
\hline 10 & & & & & & \\
\hline 11 & & & & & & \\
\hline 12 & & & & & & \\
\hline 13 & & & & & & \\
\hline 14 & & & & & & \\
\hline 15 & & & & & & \\
\hline 16 & & & & & & \\
\hline 17 & & & & & & \\
\hline 18 & & & & & & \\
\hline 19 & & & & & & \\
\hline 20 & & & & & & \\
\hline 21 & & & & & & \\
\hline 22 & & & & & & \\
\hline 23 & & & & & & \\
\hline 24 & & & & & & \\
\hline 25 & & & & & & \\
\hline 25 & & & & & & \\
\hline 26 & & & & & & \\
\hline 27 & & & & & & \\
\hline 28 & & & & & & \\
\hline 29 & & & & & & \\
\hline
\end{tabular}

Figura 2 - Protocolo para avaliação perceptivo-auditiva da voz 
Número do caso:

Nome:

Idade:

1) Quanto à fenda:

Ausência de Fenda ( ) Presença de Fenda ( )

Tipo de fenda: FP-I ( ) FTMP ( ) FP( ) FI ( ) FA ( ) FTAP( ) FFA ( ) FFAP( )

Obs.:

(Peso 2)

Nota: $(\ldots \ldots \ldots) \times 2=$

Obs.: Considerar coaptação glótica, a partir da observação da nasoendoscopia flexível.

2) Quanto à constrição supra-glótica: (vale a nota maior)

a) constrição medial: não( ) sim( ) - leve ( ) moderada ( ) severa ( )

b) constrição antero-posterior: não ( ) $\operatorname{sim}(\quad)$ - leve ( ) moderada ( ) severa ( )

Obs.:

(Peso 1)

Nota: $(\ldots \ldots \ldots) \times 1=$

3) Quanto à presença de lesão de massa em PPVV: Não ( ） Sim ( )

nódulos ( ) pólipo ( ) Edema de Reinke ( ) espessamento ( ) edema ( ) ( ) cisto

Obs.:

(Peso 2) Nota: $(\ldots \ldots \ldots) \times 2=$

4) Presença de sinais de RGE:

ausentes ( ) presentes ( ) (L) (M) (A)

hiperemia de região aritenóidea ( ) três pontos ( ) espessamento posterior ( )

reflexo nauseoso ( ) convexidade do espaço interaritenóideo ( )

Obs.:

(Peso 2) Nota: $(\ldots \ldots . .) \times 2=$.

5) Presença de sinais alérgicos em mucosa:

Ausentes ( ) presentes ( ) (L) (M) (S)

Palidez de mucosa: nasal ( ) faríngea ( )

Obs.:

(Peso 1) Nota: $(\ldots \ldots . .) \times 1=$.

Figura 3 - Protocolo para avaliação de imagens laringoscópicas 


\begin{tabular}{|c|c|c|}
\hline \multirow{2}{*}{$\begin{array}{l}\text { I - IDENTIFICAÇÃO } \\
\text { Nome: }\end{array}$} & \multirow[t]{2}{*}{ DATA: } & \multirow[t]{2}{*}{ I } \\
\hline & & \\
\hline \multicolumn{3}{|l|}{ Data de nascimento: / / / } \\
\hline \multicolumn{3}{|l|}{ Sexo: 0 ( ) feminino ( ) 1 masculino } \\
\hline \multicolumn{3}{|c|}{ II - ASPECTOS CLÍNICOS INTRA-ORAL } \\
\hline \multicolumn{3}{|l|}{ Dentes naturais: ( ) sim ( ) não } \\
\hline \multicolumn{3}{|l|}{ Presença de próteses: ( ) sim ( ) não } \\
\hline \multicolumn{3}{|c|}{$\begin{array}{l}\text { ( ) total superior ( ) total inferior ( ) parcial removível superior ( ) parcial removível inferior } \\
\text { ( ) prótese fixa unilateral ou ponte- fixa ( ) superior ( ) inferior }\end{array}$} \\
\hline \multicolumn{3}{|c|}{ Falhas dentárias: ( ) sim ( ) não } \\
\hline \multicolumn{3}{|l|}{ III - DISTÚRBIOS OCLUSAIS } \\
\hline \multicolumn{3}{|l|}{$\begin{array}{l}\text { Oclusão: } \\
\text { ( ) classe I ( ) classe II ( ) classe III }\end{array}$} \\
\hline \multicolumn{3}{|c|}{$\begin{array}{l}\text { ( ) cruzada unilateral ( ) cruzada bilateral ( ) profunda ( ) aberta anterior } \\
\text { ( ) aberta posterior ( ) desvio de linha média ( ) desproporção entre a maxila e a mandíbula }\end{array}$} \\
\hline \multicolumn{3}{|l|}{ IV - DOR À PALPAÇÃO } \\
\hline $\begin{array}{l}\text { Temporal: } \\
\text { Masseter: } \\
\text { Pterigóideo medial: } \\
\text { Esternocleidomastoideo: } \\
\text { Trapézio: } \\
\text { dor na região cervical: } \\
\text { dor na região peitoral: } \\
\end{array}$ & $\begin{array}{l}\text { ( ) } \operatorname{sim} \\
\text { ( ) } \operatorname{sim} \\
\text { ( ) } \operatorname{sim} \\
\text { ( ) } \operatorname{sim} \\
\text { ( ) } \operatorname{sim} \\
\text { ( ) } \operatorname{sim} \\
\text { ( ) } \operatorname{sim}\end{array}$ & $\begin{array}{l}\text { ( ) não } \\
\text { ( ) não } \\
\text { ( ) não } \\
\text { ( ) não } \\
\text { ( ) não } \\
\text { ( ) não } \\
\text { ( ) não }\end{array}$ \\
\hline \multicolumn{3}{|l|}{ v - SINAIS CLÍNICOS DE DTM } \\
\hline $\begin{array}{l}\text { crepitação: } \\
\text { estalido: } \\
\text { limitação do movimento mandibular: }\end{array}$ & $\begin{array}{l}\text { ( ) } \operatorname{sim} \\
\text { ( ) } \operatorname{sim} \\
\text { ( ) } \operatorname{sim}\end{array}$ & $\begin{array}{l}\text { ( ) não } \\
\text { ( ) não } \\
\text { ( ) não }\end{array}$ \\
\hline \multicolumn{3}{|l|}{ VI - HÁBITOS DELETÉRIOS } \\
\hline $\begin{array}{l}\text { bruxismo (ranger os dentes): } \\
\text { apertar os dentes: } \\
\text { roer unhas: }\end{array}$ & $\begin{array}{l}\text { ( ) } \operatorname{sim} \\
\text { ( ) } \operatorname{sim} \\
\text { ( ) } \operatorname{sim}\end{array}$ & $\begin{array}{l}\text { ( ) não } \\
\text { ( ) não } \\
\text { ( ) não }\end{array}$ \\
\hline
\end{tabular}

Figura 4 - Protocolo para avaliação odontológica 


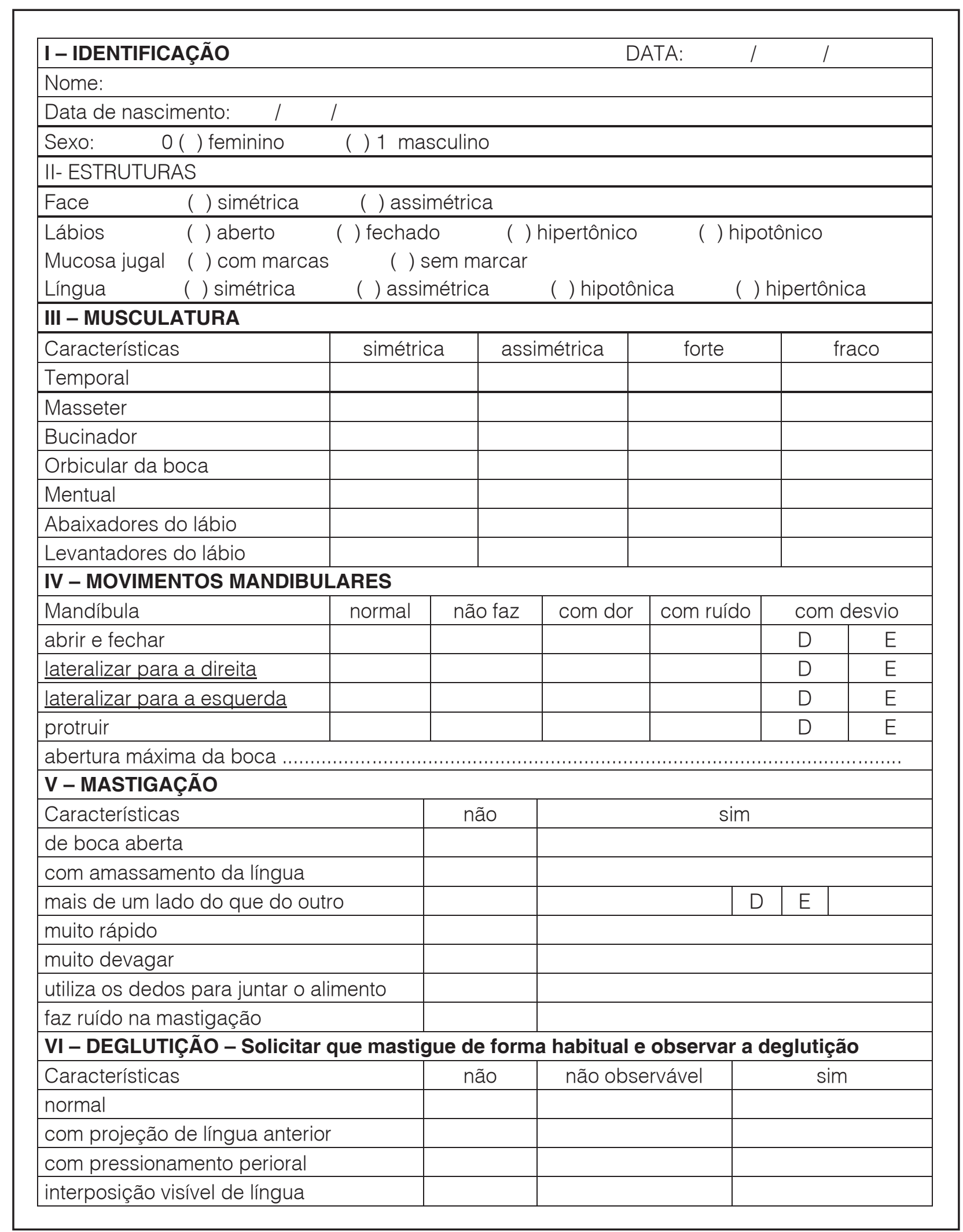

(adaptado de Bianchini, 2000)

Figura 5 - Protocolo para avaliação fonoaudiológica da motricidade orofacial 
Para a análise dos resultados foi considerado:

- Quanto às respostas ao questionário: o sujeito foi considerado com alteração vocal ou DTM quando assinalou a presença de três ou mais sintomas, segundo proposta da literatura pesquisada $33,35,26$. No caso de DTM, o sintoma de dor foi considerado indispensável;

- Para a avaliação da qualidade vocal, as amostras de fala foram analisadas por três juízas, especialistas em Voz. A voz foi considerada alterada quando, na avaliação das juízas, o professor apresentou alteração de voz em grau moderado ou severo;

- Na videonasolaringoscopia, a laringe foi considerada alterada na presença de um ou mais dos seguintes aspectos: constrição supra-glótica (constrição medial ou anterior-posterior), lesão de massa em pregas vocais (nódulos, pólipo, edema de Reinke, espessamento, edema e cisto), presença de sinais de refluxo gastroesofágico e fendas, com exceção da fenda triangular posterior;

- Nas avaliações odontológica e de motricidade orofacial, foi definido como presença de DTM sempre que se constatou ao menos três ou mais sinais, sendo imprescindível o de dor.

A pesquisa foi aprovada pelo Comitê de Ética da instituição onde foi realizada, sob o $n^{\circ}$ 137/08.

A verificação estatística constou de parte descritiva (frequência absoluta e relativa) e parte analítica com aplicação dos seguintes testes: Teste de Igualdade de Duas Proporções; Teste Exato de Fishere Índice de Concordância de Kappa.

\section{RESULTADOS}

As Tabelas 1 e 2 apresentam os dados levantados a partir do questionário e caracterizam os participantes quanto aos aspectos relacionados aos sintomas vocais (Tabela 1) e DTM (Tabela 2 e 3 ).

A Tabela 4 apresenta a descrição dos movimentos mandibulares e assimetria de face na avaliação da motricidade orofacial.

Nas tabelas seguintes foram explicitadas a comparação (Tabela 5), relação (Tabela 6) e concordância (Tabela 7) entre a avaliação de voz e DTM, autorreferida pelos professores, avaliada por fonoaudióloga, otorrinolaringologista e odontólogo.

\section{DISCUSSÃO}

Este estudo, ao verificar a presença e possível correlação entre alteração vocal e DTM em professores, avança ao propor uma análise a partir de dados de avaliação autorreferida, fonoaudiológica, otorrinolaringológica e odontológica, ou seja, numa perspectiva multidisciplinar.

Observou-se no presente estudo, associação estatística significante $(p<0,001)$ entre os professores que fizeram relato de alteração de voz e presença de três ou mais sintomas de alterações de voz.

O percentual de professores que fizeram autorreferência à alteração vocal $(82,8 \%)$ mostrou-se semelhante a estudo anteriormente realizado com o objetivo de caracterizar a voz do professor ${ }^{1}$.

Os sintomas vocais mais relatados pelos participantes deste estudo foram em ordem decrescente: rouquidão $(72,4 \%)$, perda da voz $(58,6 \%)$ e falha na voz (48,6\%). O sintoma de rouquidão tem sido relatado em diversos estudos nacionais ${ }^{6,7,9,11} \mathrm{e}$ internacionais ${ }^{3,5}$.

Sensações laringo-faríngeas também foram citadas pelos professores no presente estudo. Dentre as quais, as mais relatadas foram: cansaço ao falar $(62,1 \%)$, pigarro $(58,6 \%)$ e garganta seca $(55,2 \%)$. Essas sensações podem estar associadas ao fato dos professores fazerem uso da voz por tempo prolongado e em alta intensidade ${ }^{11}$. Parte-se da hipótese de que a sensação de cansaço ao falar pode ser provocada por tensão da musculatura orofacial, em decorrência ao uso excessivo da voz.

Em relação à DTM, as queixas autoreferidas foram mencionadas por $62,1 \%$ dos participantes. Dentre os principais sintomas de DTM, foram citados: dor de cabeça ou na face $(62,1 \%)$, presença de estalido (48,3\%), ruído na ATM $(37,9 \%)$ e travamento mandibular $(3,4 \%)$. Esses sintomas também são relatados em pesquisas anteriormente realizadas envolvendo articulação da fala e DTM 17-22,24.

Na comparação entre as propostas de avaliação de alteração de voz e DTM, foi registrada tendência à correlação significante para a aplicação do questionário. Esse dado sugere que a aplicação de questionário como o proposto nesse estudo, no qual o professor aponta seus sintomas, pode ser um instrumento importante a ser instituído com a 
Tabela 1 - Distribuição numérica e percentual das respostas dadas ao questionário que levantou aspectos relacionados a sintomas vocais $(n=29)$

\begin{tabular}{|c|c|c|c|c|c|}
\hline & \multirow{2}{*}{ VARIÁVEL } & \multicolumn{2}{|c|}{ Não } & \multicolumn{2}{|c|}{ Sim } \\
\hline & & $\mathbf{n}$ & $\%$ & $\mathbf{n}$ & $\%$ \\
\hline Alterações vocais $(n=29)$ & & 5 & 17,2 & 24 & 82,8 \\
\hline \multirow[t]{8}{*}{ Sintomas vocais $(n=29)$} & rouquidão & 8 & 27,6 & 21 & 72,4 \\
\hline & perda da voz & 12 & 41,4 & 17 & 58,6 \\
\hline & falha na voz & 15 & 51,7 & 14 & 48,6 \\
\hline & voz grossa & 19 & 65,5 & 9 & 31,0 \\
\hline & voz variando de grossa/fina & 21 & 72,4 & 8 & 27,6 \\
\hline & falta de ar & 21 & 72,4 & 2 & 27,6 \\
\hline & voz fraca & 22 & 75,9 & 7 & 24,1 \\
\hline & voz fina & 24 & 82,8 & 5 & 17,2 \\
\hline \multirow{12}{*}{$\begin{array}{l}\text { Sensações relacionadas à } \\
\text { garganta e à voz }(n=29)\end{array}$} & cansaço ao falar & 10 & 34,5 & 18 & 62,1 \\
\hline & pigarro & 12 & 41,4 & 17 & 58,6 \\
\hline & garganta seca & 13 & 44,8 & 16 & 55,2 \\
\hline & esforço ao falar & 13 & 44,8 & 16 & 51,7 \\
\hline & tosse com catarro & 16 & 55,2 & 13 & 44,8 \\
\hline & dor ao engolir & 17 & 58,6 & 12 & 41,4 \\
\hline & ardor na garganta & 18 & 62,1 & 11 & 37,9 \\
\hline & dor ao falar & 19 & 65,5 & 10 & 34,5 \\
\hline & secreção na garganta & 19 & 65,5 & 10 & 34,5 \\
\hline & areia na garganta & 23 & 79,3 & 6 & 20,7 \\
\hline & bola na garganta & 23 & 79,3 & 6 & 20,7 \\
\hline & picada na garganta & 25 & 86,2 & 4 & 13,8 \\
\hline \multirow[t]{8}{*}{ Possíveis causas $(n=29)$} & uso intensivo da voz & 9 & 31 & 15 & 51,7 \\
\hline & alergia & 15 & 51,7 & 10 & 34,5 \\
\hline & infecção respiratória & 20 & 69 & 5 & 17,2 \\
\hline & estresse & 22 & 75,9 & 5 & 17,2 \\
\hline & exposição ao barulho & 22 & 75,9 & 5 & 17,2 \\
\hline & exposição ao frio & 24 & 82,8 & 5 & 17,2 \\
\hline & infecção respiratória & 20 & 69 & 5 & 17,2 \\
\hline & gripes constantes & 24 & 82,8 & 2 & 6,9 \\
\hline
\end{tabular}

Tabela 2 - Ditribuição numérica e percentual das respostas dadas ao questionário que levantou aspectos relacionados a sintomas de DTM $(n=29)$

\begin{tabular}{|c|c|c|c|c|c|}
\hline \multirow{2}{*}{ DTM (n=29) } & \multirow{2}{*}{ VARIÁVEL } & \multicolumn{2}{|c|}{ Não } & \multicolumn{2}{|c|}{ Sim } \\
\hline & & $\mathrm{n}$ & $\%$ & $\mathbf{n}$ & $\%$ \\
\hline Sintoma de dor & dor de cabeça ou na face & 9 & 31 & 20 & 69 \\
\hline \multirow{6}{*}{$\begin{array}{l}\text { Situações em que a dor } \\
\text { ocorre }\end{array}$} & dor ao falar muito & 22 & 75,9 & 7 & 24,1 \\
\hline & dor ao acordar & 22 & 75,9 & 7 & 24,1 \\
\hline & $\begin{array}{l}\text { dor, independente do que } \\
\text { estiver fazendo }\end{array}$ & 23 & 79,3 & 6 & 20,7 \\
\hline & dor ao final do dia & 24 & 82,8 & 4 & 17,2 \\
\hline & dor ao abrir a boca & 28 & 96,6 & 1 & 3,4 \\
\hline & dor ao mastigar & 28 & 93,1 & 1 & 3,4 \\
\hline \multirow[t]{4}{*}{ Outros sintomas } & $\begin{array}{l}\text { estalido ao abrir e fechar a } \\
\text { boca }\end{array}$ & 15 & 51,7 & 14 & 48,3 \\
\hline & $\begin{array}{l}\text { ruído ao abrir e fechar a } \\
\text { boca }\end{array}$ & 18 & 62,1 & 11 & 37,0 \\
\hline & $\begin{array}{l}\text { limitação dos movimentos } \\
\text { mandibulares }\end{array}$ & 28 & 96,6 & 1 & 3,4 \\
\hline & travamento mandibular & 28 & 96,6 & 1 & 3,4 \\
\hline
\end{tabular}


Tabela 3 - Distribuição numérica e percentual das respostas dadas ao questionário à classificação da dor $(n=29)$

\begin{tabular}{llcc}
\hline DTM (n=29) & VARIÁVEL & $\mathbf{n}$ & $\%$ \\
\hline Classificação da dor & ausente & 9 & 31,0 \\
& leve & 10 & 34,5 \\
& moderada & 7 & 24,1 \\
& Grave & 3 & 10,3 \\
\hline
\end{tabular}

Tabela 4-Descrição dos movimentos mandibulares e assimetria de face na avaliação fonoaudiológica da motricidade orofacial $(n=29)$

\begin{tabular}{llcccc}
\hline & \multirow{2}{*}{ VARIÁVEL } & \multicolumn{2}{c}{ Não } & \multicolumn{2}{c}{ Sim } \\
\cline { 2 - 5 } Avaliação & & $\mathbf{n}$ & $\%$ & $\mathbf{n}$ & $\%$ \\
\cline { 2 - 5 } fonoaudiológica da MO & Desvio dos movimentos mandibulares & 13 & 44,9 & 16 & 55,1 \\
& Assimetria de face & 19 & 65,5 & 10 & 34,5 \\
& Amplitude da abertura de boca & \multicolumn{2}{c}{ Mínima } & \multicolumn{2}{c}{ Máxima } \\
& & $21,5 \mathrm{~mm}$ & \multicolumn{2}{c}{$55,16 \mathrm{~mm}$} \\
\hline
\end{tabular}

Tabela 5 - Comparação entre os percentuais referentes à alteração de voz e DTM, ausente e presente, nas avaliações autorreferida, fonoaudiológica, otorrinolaringológica e odontológica

\begin{tabular}{|c|c|c|c|c|c|c|c|c|c|}
\hline \multirow{3}{*}{ Proporção } & \multicolumn{4}{|c|}{ Ausente } & \multicolumn{4}{|c|}{ Presente } & \multirow{3}{*}{ p-valor } \\
\hline & \multicolumn{2}{|c|}{$\begin{array}{c}\text { Alteração de } \\
\text { voz }\end{array}$} & \multicolumn{2}{|c|}{ DTM } & \multicolumn{2}{|c|}{$\begin{array}{c}\text { Alteração de } \\
\text { vOz }\end{array}$} & \multicolumn{2}{|c|}{ DTM } & \\
\hline & $\mathbf{n}$ & $\%$ & $\mathbf{n}$ & $\%$ & $\mathbf{n}$ & $\%$ & $\mathbf{n}$ & $\%$ & \\
\hline autorreferida & 5 & 17,2 & 11 & 37,9 & 24 & 82,8 & 18 & 62,1 & $0,078^{\#}$ \\
\hline avaliação fonoaudiológica & 12 & 41,4 & 8 & 27,6 & 17 & 58,6 & 21 & 72,4 & 0,269 \\
\hline ORL/odontólogo & 14 & 48,3 & 10 & 34,5 & 15 & 51,7 & 19 & 65,5 & 0,286 \\
\hline
\end{tabular}

Teste de Igualdade de Duas Proporções; \# tendência a significância

Tabela 6 - Associação entre alteração de voz e DTM, para todas as avaliações: autorreferida, fonoaudiológica, otorrinolaringológica e odontológica

\begin{tabular}{llccccccc}
\hline \multirow{2}{*}{ DTM / Alteração de Voz } & \multicolumn{2}{c}{ Ausente } & \multicolumn{2}{c}{ Presente } & \multicolumn{2}{c}{ Total } & \multirow{2}{*}{ p-valor } \\
\cline { 3 - 8 } & & $\mathbf{n}$ & $\%$ & $\mathbf{n}$ & $\%$ & $\mathbf{n}$ & $\%$ & \\
\hline \multirow{2}{*}{ autorreferida } & ausente & 2 & 40 & 9 & 37,5 & 11 & 37,9 & \\
\multirow{2}{*}{ avaliação } & presente & 3 & 60 & 15 & 62,5 & 18 & 62,1 & 0,917 \\
fonoaudiológica & ausente & 6 & 50 & 2 & 11,8 & 8 & 27,6 & \\
\multirow{2}{*}{ ORL/odontólogo } & presente & 6 & 50 & 15 & 88,2 & 21 & 72,4 & $0,023^{*}$ \\
& ausente & 6 & 42 & 4 & 26,7 & 10 & 34,5 & \\
& presente & 8 & 57,1 & 11 & 73,3 & 19 & 65,5 & 0,359 \\
\hline
\end{tabular}

Teste Exato de Fisher, * diferença estatisticamente significante

Tabela 7 - Concordância entre as avaliações de voz e DTM: autorreferida, fonoaudiológica, otorrinolaringológica e odontológica

\begin{tabular}{lcc}
\hline DTM / Voz & $\%$ & p-valor \\
\hline autorreferida & $1,70 \%$ & 0,917 \\
avaliação fonoaudiológica & $41,20 \%$ & $0,023^{*}$ \\
ORL/ odontólogo & $16,30 \%$ & 0,359 \\
geral & $18,60 \%$ & $0,082^{\#}$ \\
\hline
\end{tabular}

Teste de concordância de Kappa; * diferença estatisticamente significante; \# tendência a significância 
finalidade de suspeitar-se de alterações de voz e presença de DTM. Por outro lado, tanto na análise da relação entre a presença de alterações de voz e de DTM, quanto na análise de concordância entre as avaliações, foram às avaliações fonoaudiológicas (perceptivo-auditiva da voz e de motricidade orofacial para DTM) que se mostraram significantes. A avaliação fonoaudiológica envolve aspectos subjetivos que permitem perceber e identificar as alterações. Esse dado está de acordo com a literatura ${ }^{24}$, que ao realizar a interação entre dois procedimentos, avaliação clínica e exame objetivo, constatou alta prevalência de alterações na avaliação clínica que não tiveram correlação com os achados do exame objetivo.

A relação entre alteração de voz e DTM também pode estar de acordo com achados da literatura ${ }^{25}$, que observaram presença de DTM em sujeitos com grave tensão laríngea, que fazem uso da voz profissionalmente. A princípio, pode-se supor que o uso inadequado da voz gera movimentação da musculatura orofacial e consequente desequilíbrio na ATM, com alteração da musculatura cervical, supralaríngea e dos mecanismos neuromusculares periféricos e centrais ${ }^{12-14,16}$.

$\mathrm{Na}$ literatura ${ }^{23}$, constatou-se em indivíduos com DTM, queixa de cansaço após longos períodos de fala e rouquidão. Realizados especificamente com professores, outros estudos ${ }^{6,7}$ também relacionaram alterações de voz e DTM ao desequilíbrio funcional da musculatura extrínseca da laringe, ocasionado pelas restrições dos movimentos mandibulares durante a fala.

Em relação a esses movimentos, as principais características observadas, no presente estudo, concordando com trabalhos anteriores, verificaram redução da amplitude e prevalência de desvios mandibulares durante a fala ${ }^{23,26,29}$. Conforme a literatura aponta ${ }^{31}$, essas características podem interferir na qualidade vocal.
Os achados do presente estudo confirmam os pressupostos anteriores ${ }^{26}$ que salientam que ajustes compensatórios, como redução da cavidade oral e tensão excessiva na região orofacial, são responsáveis por uma fonação com esforço e consequente queixa de cansaço, dor e desconforto ao falar muito. Nesse estudo anterior ${ }^{26}$, realizado em indivíduos com DTM, assim como na presente pesquisa, foi registrada a presença do sintoma de rouquidão.

Enquanto a aplicação do questionário mostrou tendência em constatar correlação entre queixa de alteração de voz e DTM, assim como foi verificado nas avaliações fonoaudiológicas, o mesmo não ocorreu com as avaliações realizadas pelo médico otorrinolaringologista e o odontólogo. Tal fato pode ser justificado, provavelmente, pelo fato do exame de laringe captar, de forma predominante, as alterações de fonte glótica, enquanto a correlação entre voz e DTM ${ }^{30}$ está associada na direção da alteração de filtro.

\section{CONCLUSÃO}

Os resultados apontam na direção de confirmar a presença de alteração de voz e de DTM no grupo de professores pesquisado e a existência de correlação entre esses problemas.

Os resultados obtidos vão ao encontro do objetivo do estudo, sendo que a comparação das propostas de avaliação de alteração de voz e DTM mostrou viabilidade em aplicação de questionário, no qual $82,8 \%$ dos participantes fizeram autorreferência a alteração vocal e $62,1 \%$ a DTM. As avaliações fonoaudiológicas, perceptivo-auditiva da voz e da motricidade orofacial para DTM, mostraram-se significativas tanto na análise da relação entre as alterações de voz e DTM, quanto referente à concordância entre as avaliações. 


\section{ABSTRACT}

Purpose: to check the presence and possible correlation between vocal disorders and temporomandibular joint disorders (TMJD) in teachers, from self-reported speech pathological, medical and dental evaluation data. Methods: 29 public school teachers from the city of Sorocaba SP took part in this study. The teachers filled out a questionnaire about vocal disorders and on TMJD. The four following assessments were performed: auditory-perceptive, ENT medical assessment, oral-facial mobility, and a dental assessment. The mentioning about three or more symptoms in the questionnaire determined 'presence' of vocal and TMJD complaint. Both auditory-perceptive and ENT evaluations concluded whether there was 'presence' of voice and laryngeal disorders. TMJD was considered present when three or more signs or symptoms, necessarily including pain, were registered during oral-facial mobility and dental assessments. For statistical analysis of the data the following tests were applied: Two Proportions Equality Test, Fischer's Exact Test and Kappa Agreement Test. Results: among the participating subjects, $82.8 \%$ reported having a vocal disorder, and $62.1 \%$ reported TMJD symptoms; $51.7 \%$ showed vocal disorders in ENT evaluation, and $65.5 \%$ had TMJD according to dental assessments. When comparing vocal and TMJ disorder assessments, a significant correlation was present for auditory-perceptual and oral-facial mobility for TMJD, with a tendency towards significance also when applying the questionnaire. Conclusion: the results point towards confirming the presence of TMJ and vocal disorders in the group of teachers in this research, as well as positive correlations between these two disorders.

KEYWORDS: Voice; Voice Disorders; Faculty; Temporomandibular Joint Dysfunction Syndrome; Facial Pain

\section{REFERÊNCIAS}

1. Fuess VLR, Lorenz MC. Disfonia em professores do ensino municipal: prevalência e fatores de risco. Rev. Bras. Otorrinolaringol. 2003; 69(6):807-12.

2. Roy N, Merril RM, Thibeault S, Gray SD, Smith EM. Voice disorders in teachers and the general population: effects on work performance, attendance, and future career choices. J Speech Lang Hear Res. 2004; 47(3):542-51.

3. Simberg S, Sala E, Vehmas K, Laine A. Changes in the prevalence of vocal symptoms among teachers during a twelve-year period. J Voice. 2005; 19(1):95-102.

4. Gillivan-Murphy $P$, Drinnan MJ, O'Dwyer TP, Ridha $\mathrm{H}$, Carding $\mathrm{P}$. The effectiveness of a voice treatment approach for teachers with self-reported voice problems. J Voice. 2006; 20(3):423-31.

5. Preciado-López J, Pérez-Fernández C, CalzadaUriondo M, Preciado-Ruiz P. Epidemiological study of voice disorders among teaching professionals of La Rioja, Spain. J Voice. 2007; 22(4):489-508.

6. Ferreira LP, Giannini PP, Figueira S, Silva E, Karmann DF, Souza TMT. Condições de produção vocal de professores da Prefeitura do Município de São Paulo. Rev Dist Comun. 2003; 14(2):275-307.

7. Ortiz E, Costa EA, Spina AL, Crespo NA. Proposta de modelo de atendimento multidisciplinar para disfonias relacionadas ao trabalho: estudo preliminar. Rev. Bras. Otorrinolaringol. 2004; 70(5):590-6.

8. CEREST - Centro de Referência em Saúde do Trabalhador. Distúrbios de voz relacionados ao trabalho [Boletim Epidemiológico Paulista - $n^{\circ} 26$ ]. São Paulo; 2006. [acesso em: 17 de novembro de 2008] Disponível em: URL: <http://www.cve.saude. sp.gov.br/agencia/ bepa26_dist.htm>.

9. Tavares EL, Martins RH. Vocal evaluation in teachers with or without symptoms. J Voice. 2007; 21(4):407-14.

10. Jardim R, Barreto SM, Assunção AA. Condições de trabalho, qualidade de vida e disfonia entre docentes. Cad. Saúde Pública. 2007; 23(10):2439-61 .

11. Lima MB. Sintomas vocais, alterações da qualidade vocal e laríngea em professores: análise de instrumentos. [dissertação]. São Paulo (SP): Pontifícia Universidade Católica de São Paulo; 2008.

12. Cookman S, Verdolini K. Interrelation of mandibular laryngeal functions. J Voice. 1999; 13(1):11-24.

13. Andrada e Silva MA, Bonato TRL, Costa HO. A relação entre respiração e sistema sensório motor oral em crianças disfônicas. Rev. CEFAC. 2004; 6(1):58-66. 
14. Villanueva $P$, Valezuela S, Santender H, Zunica C, Ravera MJ, Milares R. Efecto de la postura de cabeza em mediciones de la via aérea. Rev. CEFAC. 2004; 6(1):44-8.

15. Troni CR, Arakaki FN, Lima FS, Mott L, Rodrigues KA, Ferreira LP. Professores em contexto profissional e não profissional: análise objetiva e subjetiva dos aspectos da articulação e da postura. Dist Comun. 2006; 18(2):179-88.

16. Seifert E, Kollbrunner J. An update in thinking about nonorganic voice disorders. Arch Otolaryngol Head Neck Surg. 2006; 132(10):1128-32.

17. Mariz ACR, Campos PSF, Sarmento VA. Avaliação dos deslocamentos de disco da articulação temporomandibular. Braz. Oral Res. 2005; 19(1):63-8.

18. Elias FM, Birman EG, Matsuda CK, Oliveira IRS, Jorge WA. Ultrasonographic findings in normal temporomandibular joints. Braz. Oral Res. 2006; 20(1):25-32.

19. Siqueira JTT, Ching LH, Nasri C, Siqueira SRDT, Teixeira MJ, Heirs G, Valle LBS. Clinical study of patients with persistent orofacial pain. Arq. Neuropsiquiatr. 2004; 62(4):988-96.

20. Bianchini EMG, Paiva G, Andrade CRF de. Movimentos mandibulares na fala: interferência das disfunções temporomandibulares segundo índices de dor. Pró-Fono. 2007; 19(1):7-18.

21. Farella MA, Michelotti A, Bocchino T, Cimino R, Laino $\mathrm{AMH}$, Steenks MH. Effects of orthognathic surgery for class III malocclusion on signs and symptoms of temporomandibular disorders and on pressure pain thresholds of the jaw muscles. Int $\mathrm{J}$ Oral Maxillofac Surg. 2007; 36(7):583-7.

22. Felício CM, Melchior MO, Silva MAMR, Celeghini RMS. Desempenho mastigatório em adultos relacionado com a desordem temporomandibular e com a oclusão. Pró-Fono. 2007; 19(2):151-8.

23. Taucci RA, Bianchini EMG. Verificação da interferência das disfunções temporomandibulares na articulação da fala: queixas e caracterização dos movimentos mandibulares. Rev. Soc. Bras. Fonoaudiol. 2007; 12(4):274-80.

RECEBIDO EM: 20/02/2009

ACEITO EM: 10/09/2009

Endereço para correspondência:

Ilza Maria Machado

Rua Napoleão de Barros, 1058 ap. 11

São Paulo - SP

CEP: 04024-003

E-mail: ilzamachado@yahoo.com.br
24. Cozzolino FA, Rapoport A, Franzi SA, Souza RP, Pereira CAB, Dedivitis RA. Correlação entre os achados clínicos e imaginológicos nas disfunções temporomandibulares. Radiol. Bras. 2008; 41(1):13-7.

25. Van Lierde KM, De Ley S, Clement G, De Bodt $M$, Van Cauwenberge $P$. Outcome of laryngeal manual therapy in four Dutch adults with persistent moderate-to-severe vocal hyperfunction: a pilot study. J Voice. 2004; 18(4):467-74.

26. Bianchini EMG. Relações das disfunções da articulação temporomandibular com a articulação da fala. Rev Dental Press Ortodon Ortop Facial. 2000; 5(1):51-9.

27. Bianchini EMG. Avaliação fonoaudiológica da motricidade oral: distúrbios miofuncionais orofaciais ou situações adaptativas. Rev Dental Press Ortodon Ortop Facial. 2001; 6(3):73-83.

28. Demmink-Geertman L, Dejonckere $\mathrm{PH}$. Nonorganic habitual dysphonia and autonomic dysfunction. J Voice. 2002; 16(4):549-59.

29. Bianchini EMG. Paiva G, Andrade CRF. Mandibular movement patterns during speech in subjects with temporomandibular disorders and in asymptomatic individuals. Cranio. 2008; 26(1):50-8. 30. Morisso MF. Caracterização da voz de indivíduos com sintomatologia e queixa de disfunção temporomandibular. [dissertação] Santa Maria (RS): Universidade Federal de Santa Maria; 2006.

31. Silva AMT, Morisso MF, Cielo CA. Relação entre grau de severidade de disfunção temporomandibular e a voz. Pró-Fono. 2007; 19(3):279-88.

32. Ferreira LP, Giannini PP, Zenari MS. Distúrbio de voz relacionado ao trabalho: proposta de um instrumento para avaliação de professores. Dist Comun. 2007; 19:127-36.

33. Oliveira IB. Desempenho vocal do professor: avaliação multidimedimensional [tese]. Campinas (SP): Pontifícia Universidade Católica de Campinas; 1999.

34. Manfredi APS, Silva AA da, Vendite LL. Avaliação da sensibilidade do questionário de triagem para dor orofacial e desordens temporomandibulares recomendado pela Academia Americana de Dor Orofacial. Rev. Bras. Otorrinolaringol. 2001; 67(6):763-8.

35. Pordeus AMJ, Palmeira CT. Inquérito de prevalência de problemas da voz em professores da Universidade de Fortaleza. Pró-Fono. 1996; 8(2):15-24. 